\title{
Subnutrição e hipovitaminose A em pacientes com AIDS
}

\author{
Malnutrition and hypovitaminosis A in AIDS patients
}

\author{
Suzana Aparecida Silveira, José Fernando de Castro Figueiredo, \\ Alceu Jordão Junior, Maria do Rosário D. de Unamuno, \\ Maria de Lourdes Veronese Rodrigues e Hélio Vannucchi
}

\begin{abstract}
Resumo Para avaliar os estados nutricional e de vitamina $A$, foram realizadas medidas antropométricas e dosagens bioquímicas em indivíduos infectados pelo HIV-1 da região de Ribeirão Preto, SP, Brasil. O retinol plasmático foi dosado por HPLC e as reservas hepáticas de vitamina $A$ avaliadas pelo teste do RDR (relative dosis response). Os pacientes com AIDS apresentaram subnutrição significativa, com alterações mais marcantes nas medidas antropométricas relacionadas à gordura corporal, com relativa preservação dos parâmetros relacionados com a massa muscular (subnutrição marasmática). Valores de retinol plasmático

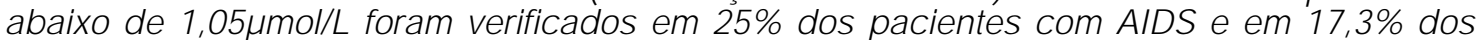
pacientes infectados pelo HIV. Valores do RDR indicativos de baixa reserva corporal de vitamina A ocorreram em $28 \%$ dos indivíduos estudados. Não houve correlação dos níveis de retinol sérico com o número de linfócitos $C D 4^{+}$, com o tempo de diagnóstico clínico de AIDS, com a PCT e o IGB.
\end{abstract}

Palavras-chaves: AIDS. Desnutrição. Hipovitaminose A. Vitamina A.

Abstract Anthropometric measurements and biochemical determinations were performed on HIV-infected individuals and on patients with AIDS from the Ribeirão Preto region, SP, Brazil, in order to evaluate their nutritional and vitamin A status. Plasma retinol was measured by HPLC, and hepatic vitamin $A$ stores were evaluated by the Relative Dose Response (RDR) test. Patients with AIDS presented significant undernutrition, with more marked alterations in the body fat compartment and a relative preservation of the parameters related to muscle mass (pattern of malnutrition predominantly of the marasmus type). Plasma retinol values below $1.05 \mu \mathrm{mol} / \mathrm{L}$ were observed in $25 \%$ of the patients with AIDS and in $17.3 \%$ of HIV-infected patients and $R D R$ values indicating low body stores of vitamin $A$ were detected in $28 \%$ of the subjects. There was no correlation between serum retinol levels and CD4 lymphocyte counts, clinical diagnosis of AIDS, low BMI or AFI. On the other hand, hypovitaminosis A status was associated with low BMI.

Key-words: AIDS. Malnutrition. Hypovitaminosis A. Vitamin A.

\footnotetext{
Divisões de Moléstias Infecciosas e Tropicais e de Nutrologia do Departamento de Clínica Médica e Departamento de Oftalmologia e Otorrinolaringologia. Faculdade de Medicina de Ribeirão Preto da Universidade de São Paulo, Ribeirão Preto, SP, Brasil.

Apoio financeiro: CNPq processo 522342/95-7.

Endereço para correspondência: Dr. José Fernando de Castro Figueiredo. Departamento de Clínica Médica, Divisão de Moléstias Infecciosas e Tropicais/Faculdade de Medicina de Ribeirão Preto. Av. Bandeirantes 3600. Campus Universitário de Monte Alegre, 14048-900, Ribeirão Preto, SP, Brasil.
}

Recebido para publicação em 21/5/98. 
Asubnutrição protéico-energética é freqüente na AIDS e pode ocorrer precocemente 2 . Apesar disso, não está clara a importância da desnutrição como fator agravante da imunodepressão e/ou acelerador da sua evolução 1422.

Em 1959, Schrimshaw et al18 descreveram associação de alterações do estado nutricional e maior susceptibilidade a infecções. A resposta imune celular e a produção de anticorpos podem apresentar anormalidades em estados de déficit nutricional6 21 . Dentre as carências nutricionais que vêm sendo implicadas na maior suscetibilidade a doenças infecciosas, estão as hipovitaminoses, incluindo-se a hipovitaminose A. Além de suas funções gerais no organismo, merece destaque o papel dessa vitamina nos processos de defesa frente às infecções. Alterações na quantidade, características e distribuição de linfócitos em órgãos linfóides têm sido relatadas na deficiência de vitamina $A 10$. $O$ retinol aumenta a função de células NK in vitro4 10 e doses suplementares de vitamina A alteram a resposta de células fagocitárias, estimulando a fagocitose e a citotoxicidade mediada por células 17 . Anormalidades da produção de células $T$ também foram descritas em casos de deficiência de retinol 19 .

Parece razoável supor, portanto, que carências nutricionais, subclínicas ou manifestas, podem contribuir para o aparecimento de alterações nos mecanismos de defesa do indivíduo infectado pelo vírus da imunodeficiência humana (HIV), favorecendo de modo significativo o desenvolvimento da imunodeficiência.

O objetivo do presente trabalho foi avaliar o estado nutricional global e os níveis de vitamina A de pacientes de uma região do Brasil com alta prevalência de AIDS. O estudo se justifica pela carência de dados sobre avaliação nutricional global e do estado da vitamina A em pacientes com AIDS no país ${ }^{15}$.

\section{CASUÍSTICA E MÉTODOS}

Casuística. Foram avaliados 73 pacientes adultos, de ambos os sexos, com sorologia positiva para HIV-1 e 20 indivíduos saudáveis, soronegativos para HIV-1, selecionados por aspectos sócio-econômicos e de ingesta alimentar semelhantes aos pacientes anti-HIV-positivos. $\mathrm{O}$ diagnóstico de infecção pelo HIV-1 foi feito através da detecção de anticorpos no soro, por método imunoenzimático (Abbot ${ }^{\circledR}$ Recombinant HIV-1/HIV-2 - IEA). Os resultados positivos por esse método foram confirmados pelo teste de aglutinação de partículas de gelatina (Serodia ${ }^{\circledR}$, Fujirebio Inc., Tokyo, Japan).

Para os fins de análise dos resultados, os pacientes infectados pelo HIV foram agrupados, respeitando-se os critérios dos $\mathrm{CDC}^{5}$, em dois grupos: o primeiro composto pelos 23 indivíduos anti-HIV-positivos com contagem de linfócitos CD4+ igual ou superior a 200 células $/ \mathrm{mm}^{3}$ e ausência de condições clínicas de AIDS (Grupo HIV) e o segundo grupo composto pelos 50 pacientes anti-HIV-positivos com contagem de linfócitos CD4+ inferior a 200 células/mm3 e/ou com condições clínicas incluídas na definição de AIDS, segundo os critérios dos CDC (Grupo AIDS). Um terceiro grupo foi constituído pelos 20 indivíduos saudáveis, anti-HIV-negativos (Grupo Controle). O trabalho foi aprovado pela Comissão de Normas Éticas e Regulamentares do Hospital das Clínicas de Ribeirão Preto. A caracterização geral dos casos acha-se expressa na Tabela 1.

Métodos. Avaliação nutricional antropométrica: Para a avaliação antropométrica foram obtidos os seguintes parâmetros: peso corporal, altura, prega cutânea tricipital (PCT), peso ideal, \% de peso ideal, índice de massa corporal (IMC), circunferência muscular do

Tabela 1 - Caracterização geral dos pacientes incluídos no estudo.

\begin{tabular}{|c|c|c|c|c|c|c|c|c|}
\hline \multirow[t]{2}{*}{ Grupos } & \multirow[t]{2}{*}{$\begin{array}{l}\text { Idade } \\
\text { (anos) }\end{array}$} & \multicolumn{2}{|c|}{$\begin{array}{c}\text { Sexo } \\
\text { (número) }\end{array}$} & \multirow{2}{*}{$\begin{array}{c}\text { Tempo de } \\
\text { HIV+ } \\
\text { (meses) }\end{array}$} & \multirow{2}{*}{$\begin{array}{c}\text { Tempo de } \\
\text { AIDS } \\
\text { (meses) }\end{array}$} & \multirow{2}{*}{$\begin{array}{l}\text { Infecções } \\
\text { anteriores } \\
\text { (número) }\end{array}$} & \multirow{2}{*}{$\begin{array}{c}\text { Linfócitos } \\
\text { totais } \\
\left(\mathrm{mm}^{3}\right)\end{array}$} & \multirow{2}{*}{$\begin{array}{c}\text { Linfócitos } \\
\text { CD4+ }^{+} \\
\left(\mathrm{mm}^{3}\right)\end{array}$} \\
\hline & & $\mathrm{M}$ & $\mathrm{F}$ & & & & & \\
\hline HIV + & $32,70 \pm 9,93$ & 9 & 14 & $27,9 \pm 21$ & - & $0,4 \pm 0,76$ & $2254 \pm 784$ & $491 \pm 156$ \\
\hline AIDS & $33,50 \pm 8,20$ & 38 & 12 & $22,0 \pm 21$ & $11,8 \pm 12,5$ & $2,14 \pm 1,14$ & $1290 \pm 633$ & $93 \pm 113$ \\
\hline Controle & $39,00 \pm 14,8$ & 3 & 17 & - & - & - & - & - \\
\hline $\mathrm{p}$ & >0,05@ & \multicolumn{2}{|c|}{$<0,05^{\#}$} & >0,05@ & - & $<0,05 @$ & $<0,05 @$ & $<0,05 @$ \\
\hline
\end{tabular}

$@$ = Tese t de Student; \# = Qui quadrado. 
braço $(\mathrm{CMB})$ e índice de gordura do braço (IGB).

Para a análise dos resultados foram considerados os valores referenciais desses parâmetros3 8112425 .

Avaliação nutricional bioquímica: A dosagem de proteínas totais e albumina no soro foi realizada por método automatizado (Roche $\left.{ }^{\circledR}\right)$ e a dosagem de creatinina urinária segundo Clark \& Thompson7. Os valores de creatinina ideal foram considerados segundo Heymsfield et al12 e o índice creatinina-altura (ICA) foi determinado pela equação creatinina urinária atual/creatinina urinária ideal $\times 100$. Os resultados do ICA foram interpretados segundo Riella 16.

Avaliação bioquímica do estado de vitamina A: A dosagem de retinol plasmático foi realizada empregando-se cromatografia líquida de alta eficiência (HPLC) segundo a técnica de Arnaud et al1, utilizando detector espectrofotométrico (Shimadzu ${ }^{\circledR} 6 \mathrm{AV}$, Japan), coluna de $25 \mathrm{~cm} x$ $0.46 \mathrm{~cm}$ (Shim-pack ${ }^{\circledR}$ CLC - ODS, Japan) e précoluna de $1 \mathrm{~cm}$ x $4 \mathrm{~mm}$ (Shim-pack ${ }^{\circledR}$ CLC - G ODS, Japan). Os reagentes utilizados foram de procedência Merck ${ }^{\circledR}$, Darmstadt, Germany, para HPLC.

Adotou-se como limite inferior da normalidade para o retinol plasmático o valor de $1,05 \mu \mathrm{mol} /$ /itro26.
Teste de dose-resposta relativa (RDR): foi realizado da seguinte maneira: após jejum noturno de 12 horas, foi obtida amostra de sangue e, a seguir, foi administrada uma dose de 450 Equivalentes de Retinol (Arovit ${ }^{\circledR}$, Roche), por via oral. Após 5 horas da administração da vitamina A foi colhida nova amostra de sangue e o índice do teste de RDR foi calculado, expresso em porcentagem, de acordo com a seguinte fórmula: RDR $=\left(R_{5}-R_{0}\right) / R_{5} \times 100$, onde $R_{5}=$ retinol plasmático obtido após 5 horas da dose oral do palmitato de retinol e $R_{0}=$ retinol plasmático de jejum. Foi considerado normal o RDR com porcentagem inferior a $20 \%$, de acordo com a padronização existente na literatura9 26 .

Análise estatística: As variáveis contínuas com distribuição normal foram expressas em termos de média e desvio padrão e o teste t de Student foi utilizado na comparação entre dois grupos. A análise de variância (ANOVA) foi empregada na comparação entre mais de dois grupos. As proporções foram comparadas pelo teste do qui-quadrado $\left(\chi^{2}\right)$ e teste exato de Fischer. As correlações entre variáveis foram estabelecidas pelo Coeficiente de Correlação de Spearman. O nível de significância estatística adotado foi de 0,05.

\section{RESULTADOS}

Os resultados das avaliações nutricionais antropométrica e bioquímica estão relacionados na Tabela 2.

O número de pacientes e respectiva porcentagem em relação ao total de casos, nos quais se observou níveis de retinol sérico abaixo de $1,05 \mu \mathrm{mol} / \mathrm{L}$ bem como de RDR $>20 \%$, estão relacionados na Tabela 3.

Foram realizados testes de correlação da contagem de linfócitos totais e CD4+ $/ \mathrm{mm}^{3} \mathrm{com}$

Tabela 2 - Valores médios das medidas antropométricas e bioquímicas nos diferentes grupos de pacientes estudados.

\begin{tabular}{|c|c|c|c|c|}
\hline & $\begin{array}{c}\text { HIVa } \\
n=23\end{array}$ & $\begin{array}{l}\text { AIDSb } \\
n=50\end{array}$ & $\begin{array}{c}\text { Controle c } \\
n=20\end{array}$ & $\mathrm{SE}^{*}$ \\
\hline Peso (kg) & $65,04 \pm 11,73$ & $58,00 \pm 12,78$ & $67,25 \pm 9,46$ & $p<0,05$ entre $a / b$ e $b / c$ \\
\hline Altura (m) & $1,65 \pm 0,07$ & $1,68 \pm 0,08$ & $1,63 \pm 0,07$ & $p<0,05$ entre $b / c$ e $b / a$ \\
\hline Peso ideal & $61,30 \pm 6,44$ & $64,90 \pm 6,31$ & $59,11 \pm 5,53$ & $p<0,05$ entre $a / c$ e $b / c$ \\
\hline Peso ideal (\%) & $105,42 \pm 19,94$ & $89,92 \pm 17,92$ & $123,54 \pm 30,17$ & $p<0,05$ entre $a / b$ e $b / c$ \\
\hline $\operatorname{IMC}\left(\mathrm{Kg} / \mathrm{m}^{2}\right)$ & $23,76 \pm 4,20$ & $20,28 \pm 3,99$ & $25,29 \pm 3,94$ & $p<0,05$ entre $a / b$ e $b / c$ \\
\hline $\operatorname{PCT}(\mathrm{mm})$ & $13,80 \pm 7,90$ & $7,55 \pm 6,03$ & $20,95 \pm 6,61$ & $\mathrm{p}<0,05$ entre todos \\
\hline $\mathrm{CMB}(\mathrm{cm})$ & $23,58 \pm 4,02$ & $22,73 \pm 3,36$ & $23,31 \pm 2,51$ & $p>0,05$ entre todos \\
\hline IGB (mm/dm²) & $1,13 \pm 0,70$ & $0,60 \pm 0,53$ & $1,69 \pm 0,54$ & $\mathrm{p}<0,05$ entre todos \\
\hline ICA & $86,54 \pm 29,67$ & $68,86 \pm 27,94$ & NR & $\mathrm{p}<0,05$ \\
\hline IMC ideal (\%) & $108,63 \pm 19,45$ & $91,12 \pm 18,14$ & $116,89 \pm 18,70$ & $p<0,05$ entre $a / b$ e $b / c$ \\
\hline PCTideal (\%) & $89,90 \pm 46,03$ & $55,14 \pm 38,11$ & $131,82 \pm 38,76$ & $p<0,05$ entre todos \\
\hline CMB ideal (\%) & $98,00 \pm 16,06$ & $91,53 \pm 13,35$ & $99,18 \pm 10,78$ & $\mathrm{p}>0,05$ entre todos \\
\hline Prot. totais (g\%) & $9,06 \pm 1,18$ & $9,21 \pm 1,40$ & NR & $p>0,05$ \\
\hline Albumina $(g \%)$ & $4,05 \pm 0,69$ & $3,99 \pm 0,82$ & NR & $p>0,05$ \\
\hline
\end{tabular}

$\overline{\mathrm{SE}}$ = significância estatística; * Teste t de Student; $\mathrm{NR}=$ não realizado 
os níveis plasmáticos de retinol de jejum e com as medidas antropométricas (IMC, PCT e IGB) obtidas nos pacientes infectados pelo HIV e nos pacientes com AIDS. Os valores de retinol plasmático de jejum foram também correlacionados com os valores das medidas antropométricas destes pacientes bem como com o tempo transcorrido desde o diagnóstico de AIDS e a dosagem sérica da vitamina A. Os resultados acham-se expressos na Tabela 4.

Tabela 3 - Freqüência de valores anormais obtidos na avaliação do estado da vitamina A, nos diferentes grupos.

\begin{tabular}{|c|c|c|c|c|c|c|c|}
\hline \multirow{3}{*}{ Parâmetro } & \multicolumn{6}{|c|}{ Grupos } & \multirow{3}{*}{ SE } \\
\hline & \multicolumn{2}{|c|}{ AIDSa } & \multicolumn{2}{|c|}{$\mathrm{HIVb}$} & \multicolumn{2}{|c|}{ Controlec } & \\
\hline & $\mathrm{n}^{\circ}$ & $\%$ & $\mathrm{n}^{\circ}$ & $\%$ & $\mathrm{n}^{\circ}$ & $\%$ & \\
\hline Retinol $<1,05 \mu \mathrm{mol} / \mathrm{L}$ & 12 & 25,0 & 4 & 17,3 & 0 & 0,0 & $>0,05(\mathrm{a} / \mathrm{b})$ \\
\hline RDR > 20\% & 11 & 28,2 & 3 & 27,6 & - & - & $>0,05$ \\
\hline
\end{tabular}

SE = significância estatística, Teste exato de Fisher.

Tabela 4 - Índices de significância estatística (p) obtidos no Coeficiente de Correlação de Spearman entre variáveis estudadas nos grupos HIV e AIDS.

\begin{tabular}{llc} 
& & Grupos \\
\cline { 2 - 3 } Correlação & HIV & AIDS \\
\cline { 2 - 3 } IMC x CD4+ & & Índice de significância estatística (p) \\
PCTx CD4+ & 0,86 & 0,05 \\
IGB x CD4+ & 0,74 & 0,76 \\
IMC x Retinol & 0,92 & 0,82 \\
PCTx Retinol & 0,79 & 0,01 \\
IGB x Retinol & 0,30 & 0,09 \\
Retinol x CD4+ & 0,32 & 0,13 \\
Retinol x Tempo de AIDS & 0,73 & 0,18 \\
\hline
\end{tabular}

\section{DISCUSSÃO}

São poucos os trabalhos na literatura que avaliaram, de maneira pormenorizada, as medidas antropométricas em pacientes infectados pelo HIV e em pacientes com AIDS. A maioria deles avaliou a perda ponderal e o IMC, sem menção de outros parâmetros 23 .

A perda de peso é freqüente na AIDS, sendo normalmente superior a $10 \%$ do peso habitual e coincide com a progressão da doença14 20 . No presente trabalho, a média do peso encontrada nos indivíduos do grupo HIV foi de $65,04 \mathrm{~kg}$, sendo de $58,00 \mathrm{~kg}$ no grupo AIDS e $67,25 \mathrm{~kg}$ no grupo Controle. Os valores porcentuais em relação ao peso ideal confirmam o deficit ponderal nos pacientes com AIDS ao revelarem média de $89,92 \%$ do peso ideal, contra índices médios de $105,42 \%$ no grupo HIV e de $123,54 \%$ no grupo Controle. Como o peso corporal guarda relação com a estatura do indivíduo, déficits ponderais como expressão de subnutrição são melhor avaliados através do IMC. No presente estudo, $78,1 \%$ dos pacientes com AIDS apresentavam IMC abaixo dos valores normais. Estes achados estão de acordo com os referidos na literatura. Kotler el al 14 verificaram redução expressiva do IMC em pacientes com AIDS ao passo que Semba et al20 encontraram valores de IMC abaixo de $19 \mathrm{~kg} / \mathrm{m}^{2} \mathrm{em} 34 \%$ dos pacientes. Essa prevalência de valores baixos de IMC explica-se, nesse estudo, pela inclusão de pacientes assintomáticos.

A PCT expressa a quantidade de gordura corporal do indivíduo. Este parâmetro mostrouse bastante alterado nos pacientes com AIDS, que apresentaram valores médios de $7,55 \mathrm{~mm}$. No grupo HIV encontramos valores de $13,80 \mathrm{~mm}$ e, no grupo controle, $20,95 \mathrm{~mm}$. Os valores médios em relação à PCT ideal foram $55,14 \%$ no grupo AIDS, $89,90 \%$ no grupo HIV e $131,82 \%$ no grupo controle.

O IGB mostrou-se também significativamente reduzido nos pacientes com AIDS em relação aos grupos HIV e Controle. Houve diferença significativa também entre o grupo HIV e Controle, tal como observado em relação à PCT. Como o IGB deriva da PCT, esse fato explica a linearidade desses resultados. 
A CMB avalia a magnitude da massa muscular corporal, dando noções quantitativas da reserva protéica do organismo. Este parâmetro esteve relativamente bem preservado nos pacientes estudados. A CMB média foi de $22,73 \mathrm{~cm}$ nos pacientes com AIDS, $23,58 \mathrm{~cm}$ no grupo HIV e $23,31 \mathrm{~cm}$ no grupo controle. Os valores médios de porcentual em relação à CMB ideal foram de $91,53 \%, 98 \%$ e $99,18 \%$ nos grupos AIDS, HIV e Controle, respectivamente, sem diferença estatística entre os mesmos.

Dessa forma, a avaliação antropométrica mostrou, de modo geral, que os pacientes com AIDS apresentavam subnutrição significativa, quando comparados com os indivíduos infectados pelo HIV e com os controles. As diferenças mais marcantes foram observadas em relação às medidas antropométricas gerais (peso e IMC) e às relacionadas à gordura corporal (PCTe IGB). A medida antropométrica relacionada à massa muscular (CMB) estava relativamente preservada. Esses resultados, em conjunto com os observados em relação à dosagem de albumina sérica e ao ICA, permitem a conclusão de que o padrão de subnutrição predominantemente encontrado na AIDS é do tipo marasmático. Não houve correlação das medidas antropométricas gerais (IMC, PCT e IGB) com a contagem de linfócitos CD4 dos pacientes dos grupos AIDS e HIV.

Com relação ao estado da vitamina $A$, verificamos valores abaixo de $1,05 \mu \mathrm{mol} / \mathrm{L}$ em $25 \%$ dos pacientes com AIDS e em 17,3\% dos infectados pelo HIV. Valores do RDR indicativos de baixa reserva corporal de vitamina $A$ foram verificados em cerca de $28 \%$ dos pacientes anti-HIV-positivos, independentemente de apresentarem ou não imunodeficiência significativa. Esses dados apontam para uma alta prevalência de hipovitaminose $A$ nos pacientes infectados pelo HIV na região de Ribeirão Preto, SP, Brasil. Os valores de retinol plasmático de jejum não se correlacionaram com a contagem de linfócitos CD4+ e nem com o tempo de diagnóstico clínico de AIDS. Por outro lado, o estado de hipovitaminose A esteve associado a baixos valores de IMC nos pacientes com AIDS, o que não se observou em relação à PCT e ao IGB.

Um aspecto de grande importância foi a verificação de baixos níveis de retinol em proporção significativa dos indivíduos infectados pelo HIV, assintomáticos e sem distúrbios aparentes de ingestão vitamínica. Perdas anormais, associadas à infecção pelo HIV13, poderiam, pelo menos em parte, justificar esses resultados.

Os dados do presente trabalho reforçam a impressão de que a hipovitaminose $A$ pode ocorrer na AIDS de forma independente do grau de desnutrição global e da intensidade da imunodepressão do paciente23. Novos estudos devem ser feitos para avaliar o impacto dessas alterações nutricionais na evolução clínica e na sobrevida dos pacientes. A esse respeito, Tang et al 22 não verificaram, em pacientes norteamericanos, associação dos níveis de retinol com a evolução da doença.

\section{REFERÊNCIAS BIBLIOGRÁFICAS}

1. Arnaud J, Fortiz I, Blachier S, Kia D, Favier A. Simultaneous determination of retinol, alpha-tocopherol and beta-carotene in serum by isocratic high-performance liquid chromatography. Journal of Chromatography 572:103-116, 1991.

2. Beach RS, Mantero-Atienza E, Shor-Posner G, Javier JJ, Szapocznik J, Morgan R, Sauberlich HE, Cornwell $\mathrm{PE}$, Eisdorfer C, Baum MK. Specific nutrient abnormalities in asymptomatic HIV-infection. AIDS 6:701-708,1992.

3. Blackburn GL, Bistrian BR, Maini BS, Schlamm HT, Smith MF. Nutrition and metabolic assessment. Journal of Parenteral and Enteral Nutrition 1:11-22, 1977.

4. Bowmann TA, Goodewardene IM, Pasatiempo AM, Ross AC, Taylor CE. Vitamin A deficiency decreases Natural Killer cells activity and interferon production in rats. Journal of Nutrition 120:1264-1273, 1990.

5. Centers for Disease Control. 1993 revised classification system for HIV infection and expanded surveillance case definition for AIDS among adolescents and adults. Morbidity and Mortality Weekly Report 41:1-3,1992.

6. Chandra RK. Micronutrients and immune functions. An overview. Annals of the New York Academy of Sciencies 587:9-15,1990.

7. Clark Jr LC, Thompson HL. Determination of creatine and creatinine in urine. Analytical Chemistry 21:12181221, 1949.

8. Frisancho AR. Triceps skin fold and upper muscle size norms for assessment of nutritional status. American Journal of Clinical Nutrition 27:1052-1058, 1974.

9. Furr HC. Training manual for assessing vitamin A status by using the modified Relative Dose Response and the Relative Dose Response assays. Washington, DC, 1992.

10. Goss GD, McBurney MW. Physiological and clinical aspects of vitamin A and its metabolites.Critical Reviews in Clinical Laboratory Sciencies 29:185-215,1992. 
11. Gurney JM, Jelliffe DB. Arm anthropometry in nutritional assessment normogram for rapid calculation of muscle circunference and cross-sectional muscle and fat areas. American Journal of Clinical Nutrition 26:912-915, 1973.

12. Heymsfield SB. Anthropometric measurement tf muscle bone-free arm area. American Journal of Clinical Nutrition 36:680-690, 1982.

13. Jordão Jr AA, Figueiredo JFC, Silveira $S$, JunqueiraFranco MVM, Vannuchi $\mathrm{H}$. Excreção urinária de vitamina A e substâncias reativas ao ácido tiobarbitúrico em pacientes com AIDS. Revista do Hospital das Clinicas da Faculdade de Medicina de São Paulo 53:11-15, 1998.

14. Kotler DP. Effect of malnutrition on the progression of AIDS. HIV-Advances in Research and Therapy 3:1723,1993.

15. Pereira PC. Impacto nutricional na Síndrome de Imunodeficiência Adquirida (SIDA): relação com o estado clínico-imunitário dos indivíduos. Tese de Doutorado, Universidade Estadual Paulista, Botucatu, SP, 1992.

16. Riella MC. Avaliação nutricional e metabólica. In: Riella MC (ed) Suporte Nutricional Parenteral e Enteral, Guanabara Koogan, Rio de Janeiro, p.19, 1985.

17. Ross AC. Vitamin A status: relationship to immunity and the antibody response. Proceedings of the Society of Experimental Biology and Medicine 200:303-320, 1992.

18. Schrimshaw NS, Taylor CE, Gordon JE. Interactions of nutrition and infection. American Journal of the Medical Sciences 237:367-403, 1959

19. Semba R, Muhilal L, Ward BJ, Griffin DE, Scott AL, Natadisastra G, West KPJr, Sommer A. Abnormal T-cell subset proportions in vitamin A-deficient children. The
Lancet 341:5-8, 1993.

20. Semba R, Caiaffa WT, Grahan NM, Cohn S, Vlahov D. Vitamin A deficiency and wasting as predictors of mortality in human immunodeficiency virus-infected injection drug users. Journal of Infectious Diseases 171:1196-1202, 1995.

21. Smith SM, Levy NS, Hayes CE. Impaired immunity in vitamin A-deficient mice. Journal of Nutrition 117:857865, 1987.

22. Tang AM, Grahan NM, Semba RD, Saah AJ. Association between serum vitamin $A$ and $E$ levels and HIV-1 disease progression. AIDS 11:613-620,1997.

23. Tassie JM, Besson J, Perez M, Berard A, Cuzin L, Marchou B, Auvergnat JC. Selenium (SE), vitamin A (VA), Vitamin E (VE) serum depletions are correlated with weight loss and not CD4-cell depletion or AIDS status. In: Abstract of XI International Conference on AIDS, Vancouver, Canada, We. p. B.-3261,1996.

24. Thomas AE, McKay DA, Cutlip MB. A normogram method for assessing body weight. American Journal of Clinical Nutrition 29:302-304, 1976.

25. Vannucchi H, Cunha DF, Dutra de Oliveira JE, Marchini JS. Arm fat index as an alternative parameter in the assessment of nutritional status of hospitalized patients. Journal of Nutritional Medicine 3:31-34,1992.

26. World Health Organization. Indicators for assessing vitamin A deficiency and their application in monitoring and evaluating intervention programes. Report for a World Health Organization/UNICEF consultation. Geneva, Switzerland, 1994. 